Fetal Diagnosis and Therapy

\title{
The Use of Amniotic Fluid Discordance in the Early Second Trimester to Predict Severe Twin-Twin Transfusion Syndrome
}

\author{
Ryo Yamamoto ${ }^{a}$ Keisuke Ishii ${ }^{a}$ Haruka Muto $^{b}$ Haruna Kawaguchi ${ }^{a}$ \\ Masaharu Murata $^{a}$ Shusaku Hayashi ${ }^{a}$ Mitsuru Matsushita ${ }^{b}$ \\ Takeshi Murakoshi ${ }^{b}$ Nobuaki Mitsuda ${ }^{a}$ \\ ${ }^{a}$ Maternal Fetal Medicine, Osaka Medical Center and Research Institute for Maternal and Child Health, Osaka,

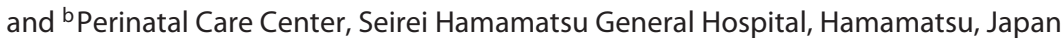

\section{Key Words}

Amniotic fluid discordance - Twin-twin transfusion syndrome - Monochorionic twin · Ultrasound screening · Second trimester

\begin{abstract}
Introduction: The appropriate effectiveness of inter-twin amniotic fluid discordance (AFD) in the early second trimester for the prediction of severe twin-twin transfusion syndrome (TTSS) was evaluated. Materials and Methods: The largest AFD between 16 and 18 weeks' gestation was analyzed in correlation with TTTS development defined by polyhydramnios with a maximum vertical pocket (MVP) $\geq 8 \mathrm{~cm}$ combined with oligohydramnios with a MVP $\leq 2 \mathrm{~cm}$ using the receiver operating characteristics curve. All pregnancies were stratified according to an AFD cutoff, and perinatal outcomes were compared between two groups. Results: A total of 223 twin monochorionic pregnancies met the inclusion criteria and 20 patients (8.9\%) developed TTTS. An AFD $\geq 4 \mathrm{~cm}$ was calculated to be the optimal point of demarcation to predict subsequent TTTS. The sensitivity and specificity of this AFD cutoff for the development of TTSS were 70 and
\end{abstract}

\section{KARGER}

(๑) 2013 S. Karger AG, Basel

$1015-3837 / 13 / 0341-0008 \$ 38.00 / 0$

E-Mail karger@karger.com

www.karger.com/fdt
$97 \%$, respectively. An AFD $\geq 4 \mathrm{~cm}$ was associated with a significantly increased risk of the development of TTTS (70 vs. $2.9 \% ; \mathrm{p}<0.01)$. Those pregnancies with AFD tended to deliver at an earlier gestational age and were also significantly associated with intrauterine fetal deaths. Discussion: The AFD between monochorionic diamniotic twins in the early second trimester may be useful for the prediction of severe TTTS development.

Copyright $\odot 2013$ S. Karger AG, Basel

\section{Introduction}

Twin-twin transfusion syndrome (TTTS) is the most critical issue in the prenatal management of a monochorionic diamniotic (MCDA) twin pregnancy and it carries a high mortality and morbidity without treatment [1]. Prenatal treatment by fetoscopic laser photocoagulation (FLP) has been shown to improve the prognosis of affected fetuses [2-4]. Therefore, the early prediction and diagnosis of severe TTTS, especially developed in the mid-second trimester, is extremely important. Several ultrasonographic findings, including inter-twin crown-rump length 
discrepancy, nuchal translucency discrepancy, and abnormal Doppler flow of the ductus venosus have been reported as first trimester predictive factors of subsequent TTTS [5-7]. However, it remains unclear whether these parameters are appropriate for its prediction [8-10].

The development of TTTS has been attributed to an imbalanced circulating plasma volume caused by the blood flow from one fetus to the other via placental anastomoses [11]. The change in the renin-angiotensin cascade induced by hypervolemia of the recipient and hypovolemia of the donor exacerbates amniotic fluid discordance [12]. The diagnosis is based on the presence of polyhydramnios of the recipient twin combined with oligohydramnios of the donor twin. Therefore, moderate AFD between MCDA twins, which do not fulfill the criteria of TTTS, could have emerged prior to the development of TTTS, and the finding could serve as a valuable predictor. Previous studies have advocated moderately discordant amniotic fluid in the second trimester as a predictor for the subsequent development of TTTS [7, 13]. However, the cutoff of AFD required to have a high predictive value has not been fully elucidated.

The objective of this study was to validate the appropriate effectiveness of AFD in the early second trimester for the prediction of TTTS.

\section{Materials and Methods}

This was a retrospective cohort study performed at two tertiary perinatal care centers in Japan. All women gave written informed consent to participate and the study protocol was approved by the ethical committee of each institution.

A total of 321 MCDAs were included between October 2008 and March 2012. Pregnancies with major congenital anomalies, chromosomal abnormalities, intrauterine fetal death (IUFD) before 15 weeks of gestation, and twin-reversed arterial perfusion were not included. Pregnancies that developed TTTS within 7 days from the first visit to our hospital were also excluded. Maternal and neonatal data from all pregnancies were collected. The diagnosis of monochorionicity was made at the first trimester ultrasound [14] and confirmed postnatally by placental examination. Serial ultrasonographic assessment, including measurement of the maximum vertical pocket (MVP) of each twin and estimated fetal weight (EFW), was performed at intervals of at least 2 weeks after 16 weeks' gestation. The AFD was calculated by subtracting the smaller MVP from the larger MVP between 16 and 18 weeks' gestation. If there was more than one AFD measurement between 16 and 18 weeks' gestation, the largest AFD before the onset of TTTS was adopted as a predictor.

The diagnosis of TTTS was made by the presence of polyhydramnios with an MVP $\geq 8 \mathrm{~cm}$ combined with oligohydramnios with an MVP $\leq 2 \mathrm{~cm}$ [2]. FLP was offered when the criteria of TTTS were met before 26 weeks' gestation. Delivery was typically planned at 37-38 weeks' gestation, absent any fetal or maternal complications.

Prediction of TTTS with Amniotic Fluid Discordance
Table 1. Maternal baseline characteristics and ultrasonographic parameters

\begin{tabular}{lc}
\hline Maternal baseline characteristics & \\
Maternal age, years & $30.7 \pm 5.0$ \\
Nulliparity & $128(57)$ \\
ART & $20(8.9)$ \\
\hline Ultrasonographic parameters & \\
Gestational age at the examination, weeks & $17(16-18)$ \\
AFD, cm & $0.8(0-7.3)$ \\
EFW of the larger fetus, g & $175(79-305)$ \\
EFW of the smaller fetus, g & $145(52-275)$ \\
EFW discordant rate $>0.25$ & $37(16)$
\end{tabular}

Data are given as means $\pm \mathrm{SD}$, median (range), or $\mathrm{n}(\%)$. ART $=$ Assisted reproductive technology.

All statistical analyses were performed using a statistical software package (Windows version 17.0; SPSS, Chicago, Ill., USA). We performed univariate analysis of the relationship between AFD, gestational age at the examination, discordant rate of EFW, and the development of TTTS using logistic regression analysis. Subsequently, multiple logistic regression analysis was performed. The discordant rate of EFW was calculated by: (larger EFW smaller EFW)/larger EFW. We constructed a receiver operating characteristics (ROC) curve to assess AFD as a predictor of subsequent TTTS. The optimal cutoff was calculated using the Youden index. All pregnancies were stratified according to an AFD cutoff. Thereafter, maternal characteristics and perinatal outcomes, including TTTS, were compared between groups. Based on the normality of the data assessed by the Shapiro-Wilk W test, continuous variables were evaluated with a Student's t or Mann-Whitney U test. Nominal variables were evaluated with Fisher's exact test. A p $<0.05$ was considered statistically significant.

\section{Results}

Fifty women referred to our clinic after 19 weeks of gestation were excluded; 5 cases with major congenital anomalies, 4 cases with a twin-reversed arterial perfusion sequence, 9 cases of IUFD before 15 weeks of gestation, 2 spontaneous abortions, and 1 artificial abortion were also excluded. There were 3 cases that developed TTTS within 7 days from the first visit to our hospital, and these cases were also excluded. A total of 223 from 247 women who met the inclusion criteria were included in the study, as 24 women were excluded due to the absence of amniotic fluid volume data.

Maternal baseline characteristics and ultrasonographic parameters are presented in table 1 . The median AFD of the 223 twins was $0.8 \mathrm{~cm}$ (range: $0-7.3$ ). Twenty pa- 
Table 2. Crude and adjusted OR estimated by logistic regression analysis for GA of ultrasonographic scan, EFW discordance, and AFD

\begin{tabular}{llllllr}
\hline Characteristics & \multicolumn{2}{l}{ Univariate analysis } & & \multicolumn{2}{l}{ Multivariate analysis } \\
\cline { 2 - 3 } & OR $(95 \% \mathrm{CI})$ & $\mathrm{p}$ & & OR $(95 \% \mathrm{CI})$ & $\mathrm{p}$ \\
\hline GA at examination & $0.87(0.49-1.54)$ & 0.63 & & - & - \\
EFW discordant rate $>0.25$ & $3.10(1.14-8.41)$ & 0.02 & & $0.54(0.12-2.30)$ & 0.40 \\
AFD & $2.34(1.75-3.12)$ & $<0.01$ & & $2.34(1.75-3.12)$ & $<0.01$ \\
\hline
\end{tabular}

$\mathrm{GA}=$ Gestational age. tients (8.9\%) developed TTTS and one of them opted for a pregnancy termination following the diagnosis of TTTS. The median gestational age of TTTS onset was 19 weeks' gestation (range: 17-35). Although spontaneous IUFDs occurred in 11 cases $(2.4 \%)$, there were no cases with demise of both fetuses.

With univariate analysis, there was a significant correlation between AFD and the development of TTTS (OR: 2.34; 95\% CI: 1.75-3.12; $\mathrm{p}<0.01$ ); however, there was no correlation between gestational age at the examination and TTTS (OR: 0.87; 95\% CI: 0.49-1.54; $\mathrm{p}=0.63$ ). The prevalence of cases with a discordant rate $>0.25$ was also significantly higher in the TTTS group by the analysis with a $\chi^{2}$ test (OR: 3.10 ; 95\% CI: $1.14-8.41 ; \mathrm{p}=0.02$ ). After multiple logistic regression analysis, the only significant variable that remained was AFD (adjusted OR: 2.34; 95\% CI: $1.75-3.12$; $\mathrm{p}<0.01$; table 2).

The ROC curve of AFD in relation to the occurrence of TTTS was constructed (fig. 1). The area under the ROC curve was 0.77 and the 90 th percentile of AFD $(3.95 \mathrm{~cm})$ appeared to be an optimal point of demarcation to predict subsequent TTTS. The sensitivity and specificity of this AFD cutoff for the development of TTTS were 70 and $97 \%$, respectively. AFD was evaluated to the first decimal place, and an AFD $\geq 4 \mathrm{~cm}$ was used to assess the relation between this cutoff and other maternal characteristics and pregnancy outcomes.

After stratification of the study group by an AFD $\geq 4 \mathrm{~cm}$, no differences in maternal age, the prevalence of nulliparity, or conception via assisted reproductive therapy were present. In the group with AFD $\geq 4 \mathrm{~cm}$, the median MVP of the recipient and donor fetus were 7.0 (range: 4-8.9) and 1.3 (range: $0-4.9$ ), respectively.

An AFD $\geq 4 \mathrm{~cm}$ was associated with a significantly increased risk of developing TTTS (70 vs. $2.9 \%$; $p<0.01$ ). Cases of TTTS occurring before 26 weeks' gestation, which made them candidates for FLP, were significantly greater in the group with an AFD $\geq 4 \mathrm{~cm}$ (table 3 ). Other adverse outcomes were more frequent in twins with an

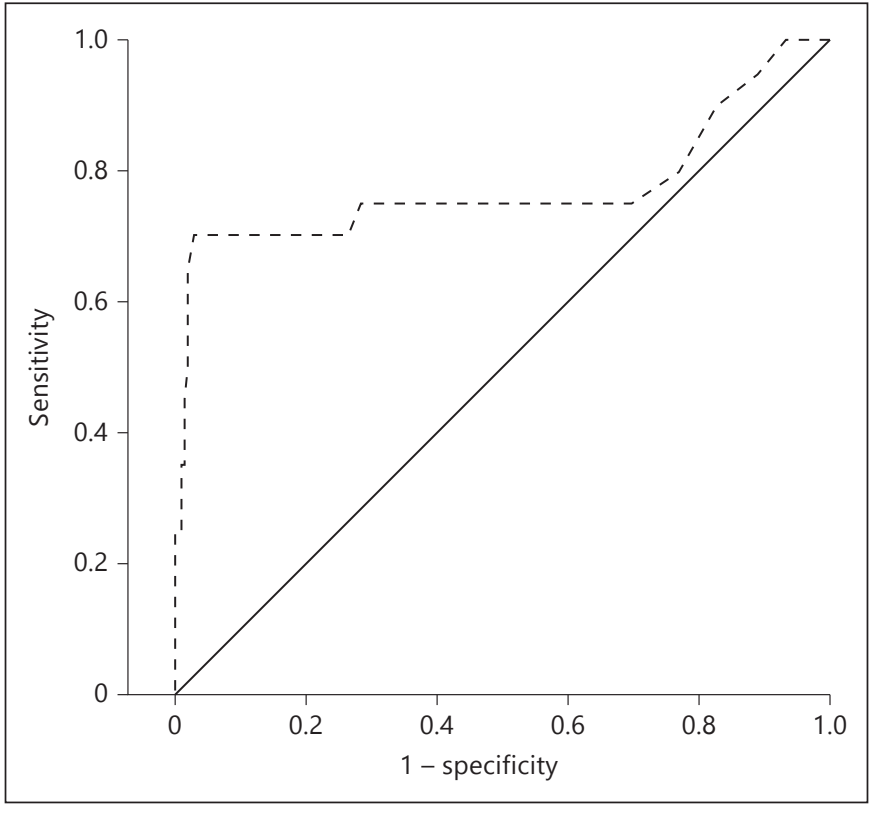

Fig. 1. ROC curve for inter-twin AFD for prediction of the development of TTTS.

$A F D \geq 4 \mathrm{~cm}$. Pregnancies with an AFD in this range tended to be delivered at an earlier gestational age and IUFD was significantly more likely to occur in twins with an AFD $\geq 4 \mathrm{~cm}$. Four of 5 IUFD cases in the group with an AFD $\geq 4 \mathrm{~cm}$ occurred after FLP was performed for TTTS. The accuracy of an AFD $\geq 4 \mathrm{~cm}$ as a predictor of TTTS is presented in table 4.

\section{Discussion}

This study found that MCDA twins presenting with moderate AFD in the early second trimester are a highrisk group for the development of TTTS. The findings of this series demonstrate that AFD in the early second tri-
10

Fetal Diagn Ther 2013;34:8-12 DOI: $10.1159 / 000348771$
Yamamoto et al. 
Table 3. Pregnancy outcomes stratified by AFD $\geq 4 \mathrm{~cm}$

Table 4. Accuracy of AFD $\geq 4 \mathrm{~cm}$ as a predictor of TTTS

\begin{tabular}{lccr}
\hline Outcome & $\begin{array}{l}\text { AFD } \geq 4 \mathrm{~cm} \\
(\mathrm{n}=20)\end{array}$ & $\begin{array}{l}\text { AFD }<4 \mathrm{~cm} \\
(\mathrm{n}=203)\end{array}$ & $\mathrm{p}$ \\
\hline Gestational age at delivery, weeks & $35(25-40)$ & $37(27-40)$ & $<0.01$ \\
Delivery at <34 weeks & $7 / 19(36)$ & $25 / 203(12)$ & 0.01 \\
TTTS & $14(70)$ & $6(2.9)$ & $<0.01$ \\
TTTS $<$ 26 weeks & $13(65)$ & $4(1.9)$ & $<0.01$ \\
Onset of TTTS, weeks & $18(17-27)$ & $23(20-35)$ & $<0.01$ \\
TOP & $1(5)$ & $0(0)$ & 0.15 \\
IUFD & $5 / 38(13)$ & $6 / 406(1.4)$ & $<0.01$ \\
\hline
\end{tabular}

Values are given as the median (range) or $\mathrm{n}(\%)$. TOP $=$ Termination of pregnancy.

\begin{tabular}{llllll}
\hline & Sensitivity & Specificity & PPV & NPV & RR (95\% CI) \\
\hline TTTS & 70 & 97 & 70 & 97 & $23.6(10.2-54.7)$ \\
TTTS $<26$ weeks & 65 & 98 & 76 & 96 & $22.5(10.3-48.8)$ \\
\hline
\end{tabular}

Values are percentages unless otherwise indicated. $\mathrm{PPV}=$ Positive predictive value; $\mathrm{NPV}=$ negative predictive value; $\mathrm{RR}=$ relative risk. mester detected $70 \%$ of subsequent TTTS in MCDA twin pregnancies, compared with only $2.9 \%$ in the low-risk group.

The prevalence of TTTS was estimated to be $8 \%$ among monochorionic twins $[15,16]$, and it is associated with high perinatal mortality and morbidity $[1,17,18]$. FLP markedly improves the prognosis of twins with TTTS in the mid-second trimester $[2-4,19]$; therefore, it is important to identify patients who should be treated with FLP in a timely manner. Additionally, some degree of discrepancy in amniotic fluid volume may indicate the onset of an imbalance of circulating plasma volume via placental anastomoses. Therefore, we considered moderate AFD in MCDA twins during the early second trimester as a predictor of TTTS and MCDA twins with moderate AFD to be at a high risk of developing TTTS. We did not include the cases that developed TTTS within 7 days from the first visit to our hospital; this was done to exclude previously existing TTTS at the time of assessment.

Lewi et al. [7] demonstrated that pregnancies with moderate AFD at 16 weeks of gestation were more likely to develop TTTS with a sensitivity of $67 \%$ and a positive predictive value of $40 \%$, even though the predictive value of assessment at this period was not necessarily high. Furthermore, the degree of AFD judged 'moderate' was not defined. Therefore, we used an AFD $\geq 4 \mathrm{~cm}$ between 16 and 18 weeks of gestation to identify the group at risk for TTTS on the basis of ROC analysis. With high specificity (97\%) and a high negative predictive value (97\%), this appears to be a cutoff that has a relatively high predictive value and indicates that it may be valuable both for identifying MCDA twin pregnancies that will not develop TTTS and for patient counseling. Once an increase of AFD above $4 \mathrm{~cm}$ was observed, a shortening of the interval of ultrasonographic assessment or a referral to the tertiary care center providing FLP for TTTS should be considered. In cases of twins that developed TTTS before 26 weeks' gestation, and whose mothers were offered to perform FLP, similar predictive values were derived. The association between moderate AFD in the early second trimester and the development of TTTS was previously described by Van Mieghem et al. [13]. Using an AFD $\geq 3.1 \mathrm{~cm}$ before 20 weeks' gestation as the predictor, sensitivity and specificity for the development of TTTS were 77 and $91 \%$, respectively. Despite the fact that the significance of the cutoff value has been debated, these predictive values were quite similar to the findings of our study.

TTTS appeared to influence the prevalence of both preterm delivery at $<34$ weeks' gestation and IUFD in the group of AFD $\geq 4 \mathrm{~cm}$. Five of 7 pregnancies that were delivered before 34 weeks' gestation in the group of 
AFD $\geq 4 \mathrm{~cm}$ developed TTTS. Spontaneous preterm delivery occurred in 4 cases after FLP, and in another case urgent delivery was performed because of TTTS onset at 27 weeks' gestation. In 5 cases with IUFD, 4 fetal deaths were also related to TTTS and another case was complicated by severe fetal growth restriction.

There may be some limitations to this study. First, because it had a retrospective cohort design, some cases were excluded due to insufficient amniotic fluid volume data. The excluded population, in which there were no cases with TTTS, was not large; thus, these exclusions appeared to have a little impact on the prediction of TTTS. Second, only AFD was analyzed as a pre- dictive factor for TTTS development in this study. About half of TTTS pregnancies are accompanied by abnormal Doppler flow in the umbilical artery, ductus venosus, or umbilical vein [20]. Therefore, a different predictive value of AFD for TTTS may be obtained when fetal Doppler parameters are included in the analysis. In view of this, a prospective cohort study should be considered.

In conclusion, the AFD between MCDA twins in the early second trimester is useful for predicting severe TTTS development. This study provides valuable information that can be used for counseling and stratification of pregnancy follow-up.

\section{References}

1 Sebire NJ, Snijders RJ, Hughes K, Sepulveda W, Nicolaides KH: The hidden mortality of monochorionic twin pregnancies. Br J Obstet Gynaecol 1997;104:1203-1207.

2 Senat MV, Deprest J, Boulvain M, Paupe A, Winer N, Ville Y: Endoscopic laser surgery versus serial amnioreduction for severe twinto-twin transfusion syndrome. N Engl J Med 2004;351:136-144.

-3 Rossi AC, D'Addario V: Laser therapy and serial amnioreduction as treatment for twintwin transfusion syndrome: a metaanalysis and review of literature. Am J Obstet Gynecol 2008;198:147-152.

-4 Sago H, Hayashi S, Saito M, Hasegawa H, Kawamoto H, Kato N, Nanba Y, Ito Y, Takahashi Y, Murotsuki J, Nakata M, Ishii K, Murakoshi $\mathrm{T}$ : The outcome and prognostic factors of twin-twin transfusion syndrome following fetoscopic laser surgery. Prenat Diagn 2010;30:1185-1191.

5 Kagan KO, Gazzoni A, Sepulveda-Gonzalez G, Sotiriadis A, Nicolaides KH: Discordance in nuchal translucency thickness in the prediction of severe twin-to-twin transfusion syndrome. Ultrasound Obstet Gynecol 2007; 29:527-532.

6 Matias A, Montenegro N, Loureiro T, Cunha M, Duarte S, Freitas D, Severo M: Screening for twin-twin transfusion syndrome at 11-14 weeks of pregnancy: the key role of ductus venosus blood flow assessment. Ultrasound $\mathrm{Ob}$ stet Gynecol 2010;35:142-148.

7 Lewi L, Lewi P, Diemert A, Jani J, Gucciardo L, Van Mieghem T, Done E, Gratacos E, Huber A, Hecher K, Deprest J: The role of ultrasound examination in the first trimester and at 16 weeks' gestation to predict fetal complications in monochorionic diamniotic twin pregnancies. Am J Obstet Gynecol 2008;199: 493, e491-e497.

8 Memmo A, Dias T, Mahsud-Dornan S, Papageorghiou AT, Bhide A, Thilaganathan B: Prediction of selective fetal growth restriction and twin-to-twin transfusion syndrome in monochorionic twins. BJOG 2012;119:417421.

9 Sperling L, Kiil C, Larsen LU, Brocks V, Wojdemann KR, Qvist I, Schwartz M, Jorgensen C, Espersen G, Skajaa K, Bang J, Tabor A: Detection of chromosomal abnormalities, congenital abnormalities and transfusion syndrome in twins. Ultrasound Obstet Gynecol 2007;29:517-526.

10 El Kateb A, Nasr B, Nassar M, Bernard JP, Ville Y: First-trimester ultrasound examination and the outcome of monochorionic twin pregnancies. Prenat Diagn 2007;27:922-925.

-11 Diehl W, Hecher K, Zikulnig L, Vetter M, Hackeloer BJ: Placental vascular anastomoses visualized during fetoscopic laser surgery in severe mid-trimester twin-twin transfusion syndrome. Placenta 2001;22:876-881.

12 Tchirikov M: Monochorionic twin pregnancy: screening, pathogenesis of complications and management in the era of microinvasive fetal surgery. J Perinat Med 2010;38:451-459.

13 Van Mieghem T, Eixarch E, Gucciardo L, Done E, Gonzales I, Van Schoubroeck D, Lewi L, Gratacos E, Deprest J: Outcome prediction in monochorionic diamniotic twin pregnancies with moderately discordant amniotic fluid. Ultrasound Obstet Gynecol 2011; $37: 15-21$.
14 Sepulveda W, Sebire NJ, Hughes K, Odibo A, Nicolaides KH: The lambda sign at 10-14 weeks of gestation as a predictor of chorionicity in twin pregnancies. Ultrasound Obstet Gynecol 1996;7:421-423.

15 Acosta-Rojas R, Becker J, Munoz-Abellana B, Ruiz C, Carreras E, Gratacos E: Twin chorionicity and the risk of adverse perinatal outcome. Int J Gynaecol Obstet 2007;96:98102 .

16 Nakayama S, Ishii K, Kawaguchi H, Hayashi S, Hidaka N, Murakoshi T, Mitsuda N: Perinatal outcome of monochorionic diamniotic twin pregnancies managed from early gestation at a single center. J Obstet Gynaecol Res 2012;38:692-697.

17 Saunders NJ, Snijders RJ, Nicolaides KH: Therapeutic amniocentesis in twin-twin transfusion syndrome appearing in the second trimester of pregnancy. Am J Obstet Gynecol 1992; 166:820-824

18 Gonsoulin W, Moise KJ Jr, Kirshon B, Cotton DB, Wheeler JM, Carpenter RJ Jr: Outcome of twin-twin transfusion diagnosed before 28 weeks of gestation. Obstet Gynecol 1990;75: 214-216.

19 Huber A, Diehl W, Bregenzer T, Hackeloer BJ, Hecher K: Stage-related outcome in twintwin transfusion syndrome treated by fetoscopic laser coagulation. Obstet Gynecol 2006;108:333-337.

20 Chmait RH, Kontopoulos EV, Korst LM, Llanes A, Petisco I, Quintero RA: Stage-based outcomes of 682 consecutive cases of twintwin transfusion syndrome treated with laser surgery: the USFetus experience. Am J Obstet Gynecol 2011;204:393, e1-6. 